\title{
Zero-lag synchronization and bubbling in delay-coupled lasers
}

\author{
J. Tiana-Alsina, ${ }^{1}$ K. Hicke, ${ }^{2}$ X. Porte, ${ }^{2}$ M. C. Soriano, ${ }^{2}$ M. C. Torrent,,${ }^{1}$ J. Garcia-Ojalvo, ${ }^{1}$ and I. Fischer ${ }^{2}$ \\ ${ }^{1}$ Departament de Física i Enginyeria Nuclear, Universitat Politècnica de Catalunya, Campus de Terrassa, Edif. GAIA, \\ Rambla de Sant Nebridi s/n, Terrassa E-08222 Barcelona, Spain \\ ${ }^{2}$ Instituto de Física Interdisciplinar y Sistemas Complejos, IFISC (UIB-CSIC), Campus Universitat de les Illes Balears, \\ E-07122 Palma de Mallorca, Spain
}

(Received 4 October 2011; published 21 February 2012)

\begin{abstract}
We show experimentally that two semiconductor lasers mutually coupled via a passive relay fiber loop exhibit chaos synchronization at zero lag, and study how this synchronized regime is lost as the lasers' pump currents are increased. We characterize the synchronization properties of the system with high temporal resolution in two different chaotic regimes, namely, low-frequency fluctuations and coherence collapse, identifying significant differences between them. In particular, a marked decrease in synchronization quality develops as the lasers enter the coherence collapse regime. Our high-resolution measurements allow us to establish that synchronization loss is associated with bubbling events, the frequency of which increases with increasing pump current.
\end{abstract}

DOI: 10.1103/PhysRevE.85.026209

PACS number(s): 05.45.Xt, 05.45.Jn, 42.65.Sf

\section{INTRODUCTION}

Chaos synchronization is a prevalent phenomenon in coupled nonlinear systems [1,2]. Because coupling signals travel at finite speeds, synchronization tends to arise at a nonzero lag. This occurs for instance in two mutually coupled semiconductor lasers [3]. Zero-lag (isochronous) synchronization despite coupling delays can be induced in different ways, e.g., by adding self-feedback to the two coupled systems [4], driving them with a third common oscillator [5], or using a relay element between them. This relay can either be active (i.e., with its own dynamics, like a third laser) [6,7] or passive (like a semitransparent mirror in the laser case) [8,9].

Much effort has been devoted to observe and quantify chaos synchronization. In particular, the effect of coupling topology on the synchronization properties of coupled oscillators has been thoroughly studied [10-15]. It has been shown, for instance, that symmetric values of coupling (and feedback) strengths and delay times favor synchronization $[12,13]$. One of the main reasons of the interest in chaos synchronization, especially in lasers, is the possibility to use this phenomenon as a method for chaos-based communication schemes and key-exchange protocols [8,16-19]. Message recovery in such schemes depends on synchronization of the communicating lasers, as recovery is not possible from a nonsynchronized state.

Some studies have quantitatively addressed the question of how synchronization is lost in coupled chaotic systems under the influence of noise or as control parameters are varied [20-25]. However, studies that investigate the mechanisms behind the loss of synchronization are rare, especially from an experimental viewpoint. Parameter changes frequently modify the underlying dynamics of the coupled elements, and these alterations are bound to affect the way synchronization is lost. There are two major mechanisms for synchronization loss of nearly identical coupled oscillators: Transverse instability of the synchronization manifold due to a blow-out bifurcation [20,21] and attractor bubbling [21-23,25]. A transition through a blow-out bifurcation is characterized by intermittent desynchronization events, a behavior that is called on-off intermittency $[23,26,27]$. Below the blow-out bifurcation there exists a transition in which invariant sets in an otherwise transversely stable synchronization manifold lose their transverse stability. This so-called bubbling bifurcation is present when varying a key parameter of the coupled system, often the coupling strength. The bubbling regime exhibits intermittent desynchronization events, similar to on-off intermittency.

Bubbling events are induced by noise and/or parameter mismatch and, as mentioned above, can be attributed to transversely unstable periodic orbits embedded in the stable synchronization manifold [22,25,28]. The local instability forces the system's trajectory to temporarily leave the synchronization manifold until resynchronization occurs. In the case studied here, bubbling is predominantly induced by unstable antimodes. Delay-coupled Stuart-Landau oscillators and Kuramoto oscillators exhibit similar saddle points [29], and thus similar behavior can be expected. Bubbling has been found in several different types of system: lasers [23,30], electronic circuits [31,32], biological cells [33], and different generic oscillators (see, e.g., Ref. [22]).

In this work we intend to connect the overall synchronization quality with the occurrence of desynchronization events attributed to the bubbling phenomenon. Our quantitative experimental approach uses two mutually delay-coupled semiconductor lasers exhibiting zero-lag synchronization as a model system. In the absence of coupling the lasers have a stable intensity output. Chaotic behavior of the lasers is induced by time-delayed feedback [34] and/or delayed mutual coupling of the lasers [3,35]. Instantaneous coupling may also lead to instabilities [36]. High-temporal-resolution measurements will reveal that the loss of synchronization, which arises as the lasers are pumped further away from threshold, is due to bubbling and will help us uncover a relation between the character of the bubbling and the type of chaotic regime exhibited by these lasers. A previous theoretical work by some of us [25] has shown that for rather small coupling strengths transverse instability exists in the system considered here. For larger coupling strengths the attractor remains transversely stable, but shows bubbling. Since we focus on bubbling as a desynchronization mechanism, we therefore choose a comparably large coupling during our experimental studies. 

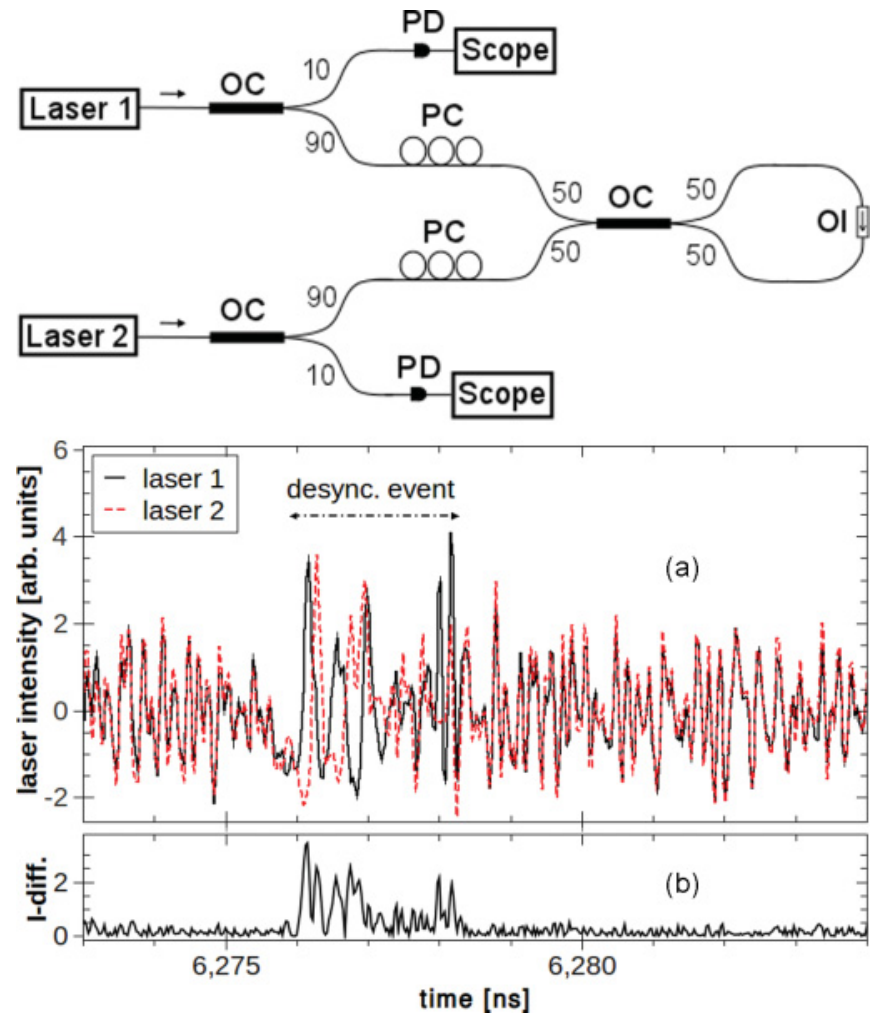

FIG. 1. (Color online) Schematic experimental setup (top panel). PC: polarization controller, PD: fast photodetector, OC: optical coupler, OI: optical isolator. Lower panel: (a) Experimental time series of synchronized fast intensity dynamics in the coherence collapse regime. A short desynchronization event is highlighted. The pump current corresponds to $1.25 I_{\mathrm{thr}}$, with $I_{\mathrm{thr}}$ being the solitary lasing threshold. (b) Corresponding normalized intensity difference (synchronization error).

The observed desynchronization behavior in our experiment is in agreement with the theoretical findings [25].

\section{EXPERIMENTAL SETUP}

Our experimental setup is depicted in Fig. 1. The fiber-based setup consists of two similar discrete mode semiconductor lasers (Eblana Photonics), operating at a nominal wavelength of $\lambda \approx 1540 \mathrm{~nm}$ and coupled symmetrically via a relay fiber loop. This loop functions as a semitransparent mirror, accounting for symmetric feedback and coupling with equal delay times. This coupling relay leads to chaotic behavior in both lasers, as well as to isochronal synchronization of their outputs. Due to the 50/50 optical coupler used to combine both laser outputs in the loop, we have identical feedback and coupling strengths. The absence of asymmetries in the coupling provides near-optimal synchronization conditions $[12,13]$. By autocorrelation analysis the feedback and coupling delay in our setup was determined to be $\tau=73 \mathrm{~ns}$. The chosen coupling strengths ensure that we avoid the regime of transverse instability due to a blow-out bifurcation [25].

The spectral characteristics of the two lasers were adjusted by tuning their temperature, in order to ensure zero spectral detuning (i.e., maximum overlap of the optical spectra). The laser temperatures and pump currents were controlled by a Thorlabs PRO8000 laser controller with accuracies $\Delta T=$ $\pm 0.01{ }^{\circ} \mathrm{C}$ and $\Delta I_{p}= \pm 0.01 \mathrm{~mA}$. Polarization controllers were used to adjust the polarization for polarization-maintained feedback and optimum coupling. An optical isolator ensured a single propagation direction in the fiber loop, to avoid interference effects. We measured the laser outputs by using fast Miteq Dr-125G-A photodetectors with $13 \mathrm{GHz}$ bandwidth, whose outputs were recorded by a LeCroy WaveMaster $816 \mathrm{Zi}$ oscilloscope with an analog bandwidth of $16 \mathrm{GHz}$ and a sampling rate of $40 \mathrm{GS} / \mathrm{s}$. With such a high time resolution we can resolve the fast dynamics on a picosecond time scale, and observe and distinguish very short intervals of synchronization or unsychronized behavior with unprecedented detail. Because both lasers are very well matched, we neglect parameter mismatch or detuning as the principal reason for bubbling.

\section{ZERO-LAG SYNCHRONIZATION}

We analyze the synchronization behavior of the coupled lasers in a current range of $I_{p}=12-17 \mathrm{~mA}$, which corresponds in our case to $\sim 1.0-1.5$ times the solitary lasing threshold $I_{\text {thr. }}$. With our setup we are able to achieve high-quality zero-lag synchronization, as shown in the high-resolution time traces of Fig. 1(a), corresponding to the coherence collapse regime. The plot depicts near-perfect synchronization, with one distinct short desynchronization event (bubbling event) clearly visible in the intensity difference (i.e., synchronization error) [Fig. 1(b)].

In the current range studied here the coupled system may exhibit two different dynamical regimes [37]: low-frequency fluctuations (LFFs) and fully developed coherence collapse (CC). The LFF regime is characterized by a slow time scale associated with the global dynamics on the mode ellipse. It manifests itself in the intensity dropouts, followed by a subsequent intensity buildup until the next dropout. The dropouts coincide with large spectral jumps toward the vicinity of the solitary laser mode. During the buildup process, the laser's dynamics drift through several optical modes. This chaotic itineracy almost monotonically tends toward higher intensities [38]. The dropout occurs when the laser's trajectory gets close to the stable manifold of an antimode, which corresponds to a saddle point [39]. This antimode also corresponds to a transversely unstable mode of the laser's chaotic attractor. In the $\mathrm{CC}$ regime, in contrast, the global dynamics occurs on a faster time scale. The dynamics of the laser in the CC regime exhibits more frequent critical events with transversely unstable antimodes, and thus more frequent subsequent bubbling events. Here we compare the different synchronization dynamics in the two regimes.

We can quantify the degree of synchronization between the two time series by using the cross-correlation at zero lag $C=$ $\frac{\left\langle\left[I_{1}(t)-\left\langle I_{1}\right\rangle\right]\left[I_{2}(t)-\left\langle I_{2}\right\rangle\right]\right\rangle}{\sqrt{\left\langle\left[I_{1}(t)-\left\langle I_{1}\right\rangle\right]^{2}\right\rangle\left\langle\left[I_{2}(t)-\left\langle I_{2}\right\rangle\right]^{2}\right\rangle}}$, where $\langle\cdot\rangle$ denotes time averaging. To further account for the fast dynamical fluctuations we integrate and normalize the synchronization error over shifting windows of one delay time $\tau$ and calculate its mean value $\chi=\left\langle\frac{1}{\tau} \sum_{\tau} \frac{\left|I_{1}-I_{2}\right|}{\left\langle I_{1}+I_{2}\right\rangle}\right\rangle$. These quantifiers are shown in Fig. 2 for increasing applied currents of the two lasers (equal currents, keeping zero spectral detuning). As the currents are increased, 


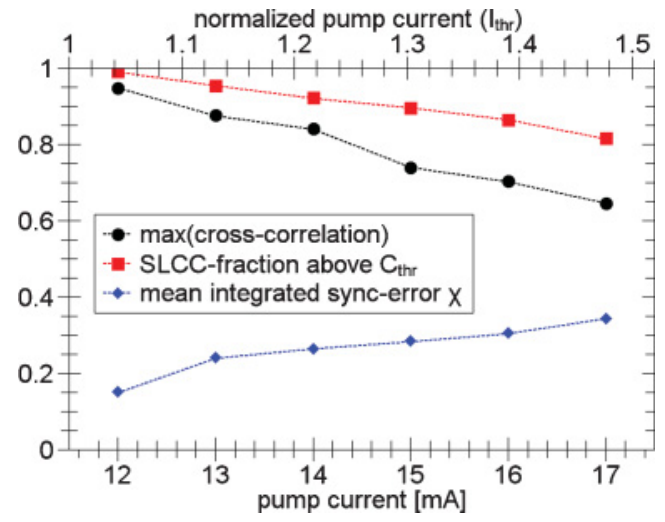

FIG. 2. (Color online) Cross-correlation at zero lag (black circles), fraction of the sliding cross-correlation above the correlation threshold of $C_{\mathrm{thr}}=0.5$ (red squares), and mean integrated synchronization error $\chi$ (blue diamonds), respectively, vs the applied pump current and the pump current normalized with respect to $I_{\text {thr }}$.

the zero-lag cross-correlation (black circles) decreases, and correspondingly the mean integrated synchronization error (blue diamonds) increases, almost linearly. In what follows we intend to understand why synchronization deteriorates with increasing pump current and how that loss is related to the change of dynamical regime (from low-frequency fluctuations for $I_{p} \sim 12 \mathrm{~mA}$ to coherence collapse for $I_{p} \sim 17 \mathrm{~mA}$ ). Figure 1(b) shows that the instantaneous synchronization error is subject to short bursts that correspond to desynchronization events. To account for the synchronization dynamics, we calculate a sliding-window cross-correlation (SLCC), in which the standard cross-correlation coefficient at zero lag is calculated over a shifting window of $1 \mathrm{~ns}$ width, and advance the window by four sampling steps ( $0.1 \mathrm{~ns})$ at a time. In that way we obtain a time trace of (local) cross-correlation values. A significant drop of the sliding cross-correlation (below a threshold $C_{\text {thr }}$ ) means that a desychronization event has occurred. We can then quantify the fraction of synchronized dynamics with respect to the total length of the SLCC time series. This fraction decreases with pump current, similarly to the zero-lag cross correlation, as shown in Fig. 2.

\section{SYNCHRONIZATION LOSS DUE TO BUBBLING}

In the LFF regime, intensity dropouts occurring simultaneously in the two lasers can be interpreted as purely deterministic events, arising from the dynamics of the coupled system. In some cases, on the other hand, noise can lead to a dropout in only one laser. Then synchronization is lost, and the other laser is affected by the dropout only after the propagation delay $\tau$, then undergoing a dropout itself. After this second event, the lasers resynchronize [3]. Figures 3(a) and 3(b) depict a time trace that shows one such desynchronization episode. The figure also compares the long-term behavior of the sliding cross-correlation [Fig. $3(c)$ ] with the corresponding output intensity time traces [Fig. 3(a)] in the regime of low-frequency fluctuations. The figure shows that the desynchronization events in the LFF regime indeed coincide with intensity dropouts at the end

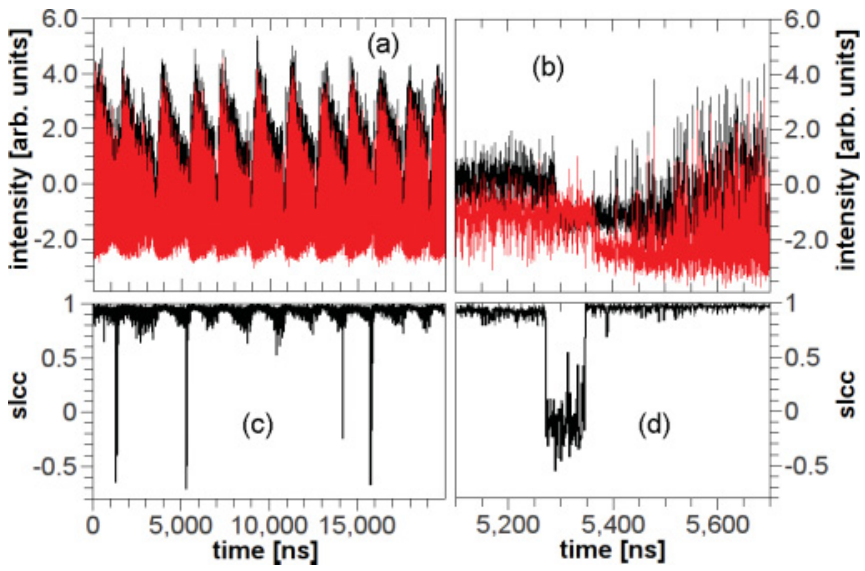

FIG. 3. (Color online) Synchronization of LFF dynamics. Output intensity time series of the two lasers $(a, b)$ and corresponding sliding cross-correlation (c, d) for a long time interval ( $a, c)$ and a magnification in time (b, d). The intensity time series were vertically shifted for better visibility. The applied pump current was $I_{p}=12 \mathrm{~mA}$, which is closely above the solitary thresholds of both lasers.

of each LFF cycle, in agreement with previous numerical results [25]. As mentioned above, the dropouts of both lasers usually occur with a relative time shift of $\tau$, resulting in desynchronization events of that length [Fig. 3(d)]. Between the desynchronization events the synchronization level is very high, with SLCC being higher than 0.95 in our experiments.

In the case of coherence collapse, which arises for higher pump currents, the synchronization dynamics are very different, due to the fact that the overall dynamics differ substantially from the LFF behavior [Fig. 4(a)]. In particular, there is no slow time scale like the one associated with the power dropouts in the LFF regime. Concurrently, the ejections of the trajectory due to unstable antimodes are more frequent and much shorter than in the LFF case, with a duration of the order of $1 \mathrm{~ns}$. The desynchronization events are associated

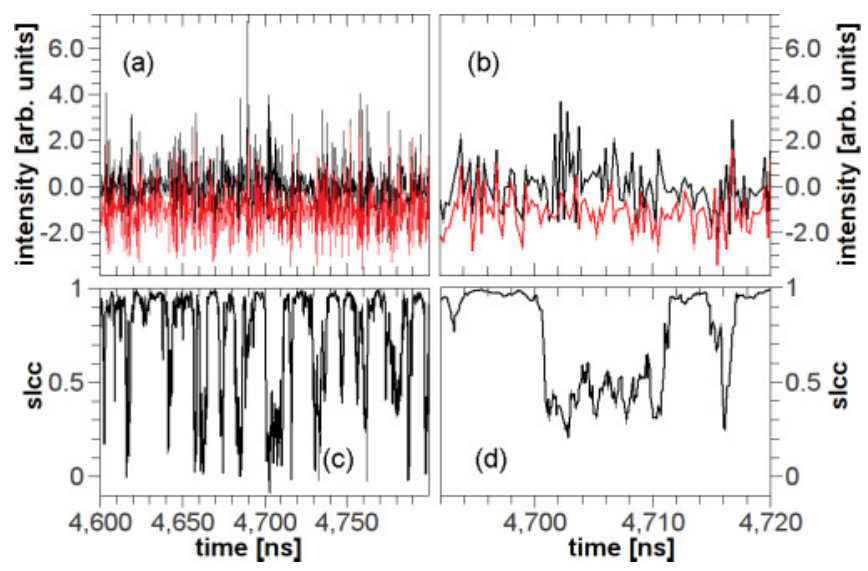

FIG. 4. (Color online) Synchronization in the coherence collapse regime. Output intensity time series of the two lasers $(a, b)$ and corresponding sliding cross-correlation $(c, d)$ for a long time interval $(a, c)$ and a magnification in time $(b, d)$. The intensity time series were vertically shifted for better visibility. The pump current was $I_{p}=16 \mathrm{~mA} \approx 1.3 I_{\mathrm{thr}}$. 


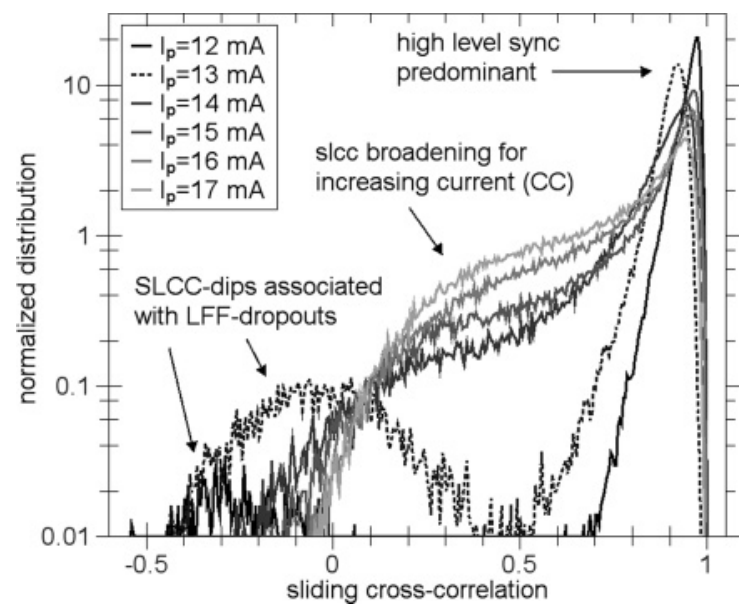

FIG. 5. Normalized distributions of the sliding cross-correlation coefficients for six different pump currents. The histograms have 401 bins.

with the ejections and therefore have a comparable duration and the same frequency [Figs. 4(c) and 4(d)]. The intervals of synchronized behavior also exhibit, as in the LFF regime, a high correlation with values of SLCC higher than 0.95. The high temporal resolution of the measurements allows us to resolve the fast synchronization dynamics with good accuracy [Figs. 4(b) and 4(d)]. The results shown in Figs. 3 and 4 allow us to infer that the average deterioration of synchronization that is observed with increasing pump current (Fig. 2) is due to an increase in the frequency of desynchronization (bubbling) events. The value of the SLCC (measuring the instantaneous correlation) during synchronized time intervals, however, does not change much with increasing current. This is quantified in Fig. 5, which shows the normalized SLCC distribution for six pump current values. The normalization is done such that the integral over all 401 bins equals 1 . The histograms cluster in two qualitatively different groups, being bimodal for the low currents corresponding to the LFF regime (12 and $13 \mathrm{~mA}$ ) and characterized by a single, broad and asymmetric peak for higher currents. The lower peaks in the SLCC distributions for the two LFF cases (12 and $13 \mathrm{~mA}$ ) are dips to negative correlation that occur at the LFF-power dropouts. As for the coherence collapse regime, although the distributions broaden significantly with increasing current, they still clearly peak at a correlation close to 1 . Consequently we conclude that intervals of high-level synchronization still occur even for large pump currents: A global decrease in synchronization would shift the distribution maxima in Fig. 5 toward lower correlation values. This conclusion is supported by calculating the fraction of time, during which synchronized dynamics persists, as depicted in Fig. 2. We chose $C_{\text {thr }}=0.5$ as synchronization threshold for the SLCC, guided by the bimodal LFF distributions of Fig. 5. Even though this value is chosen arbitrarily, a different threshold changes the slope of the curve only slightly, but the overall monotonic behavior persists.

\section{BUBBLING STATISTICS}

Given that the correlation level within the synchronized intervals does not diminish with the pump current, but the
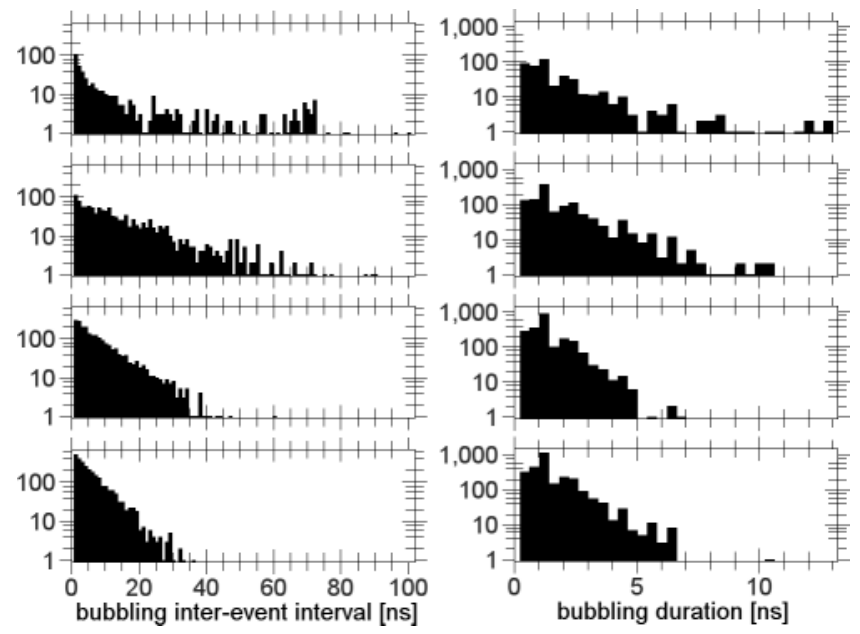

FIG. 6. Histograms of the interevent intervals (left) and the bubbling duration (right) for pump currents $I_{p}=14-17 \mathrm{~mA}$. A bubbling event is considered to occur when the SLCC decreases below a value $C_{\mathrm{thr}}=0.5$. We also introduce a threshold for a minimum event duration $\Delta T_{\mathrm{thr}}=0.5 \mathrm{~ns}$, and a minimum interevent interval $\mathrm{IEI}_{\mathrm{thr}}=0.5 \mathrm{~ns}$ below which two isolated desychronization events are considered part of a single, longer one.

average synchronization quality does, the bubbling events must become more frequent as the dynamics transitions from the LFF to the CC regime. This can already be inferred from a comparison between Figs. 3(c) and 4(c), but a more systematic quantification is needed. To that end, we now statistically quantify the duration of bubbling events and the time between consecutive events (interevent intervals [IEIs]). Figure 6 shows the corresponding histograms for $I_{p}=14-17 \mathrm{~mA}$. For input currents $I_{p}=12 \mathrm{~mA}$ and $I_{p}=13 \mathrm{~mA}$, which result in LFF dynamics, only 7 and 20 events were captured, respectively. As mentioned above, these are usually relatively long events ( $\sim \tau$ in duration) which are isolated, the IEIs being of the order of microseconds. Therefore, we present only bubbling histograms for the $\mathrm{CC}$ regime.

The IEI distributions shown in the left panels of Fig. 6 reveal that bubbling events become more closely spaced (the distribution decays faster to 0 ) with increasing current; relatively isolated events become continuously more rare with increasing $I_{p}$. The bubbling durations (right panels) are not much affected by a change in current. Nevertheless, for increasing current, fewer of the longer events are captured. The enhancement of a bubbling duration of $1 \mathrm{~ns}$ can be considered a numerical artifact, caused by the choice of a window size of $1 \mathrm{~ns}$ for the computation of the SLCC.

\section{CONCLUSION}

In summary, we have experimentally achieved high quality zero-lag synchronization of two mutually injected fibercoupled semiconductor lasers, with correlation coefficients higher than 0.95 in the synchronized regime. Our highresolution measurements have revealed general differences between the synchronization dynamics of the low-frequency fluctuation and the coherence collapse dynamical regimes. 
Using a sliding cross-correlation measurement, we have identified distinct desychronization events, which are interpreted as bubbling events. The bubbling phenomenon is seen to be responsible for the decline of synchronization with increasing current, with the desynchronization events becoming more frequent, especially with the transition from LFF to the $\mathrm{CC}$ regime. Meanwhile, the synchronized intervals maintain their high correlation level. In that way, our measurements associate, via bubbling, synchronization loss with changes in the underlying chaotic dynamics of the coupled system.

Excursions away from the synchronization manifold are detrimental to all application schemes relying on synchronization. This applies especially to chaotic optical communication. Noise-induced desynchronization events will strongly affect the efficiency of bidirectional schemes and thus must be taken into consideration for applications. Our results may therefore be helpful for future studies of these chaos communication concepts and key exchange protocols.

\section{ACKNOWLEDGMENTS}

This research was partially supported by MICINN (Spain) under projects DeCoDicA (TEC2009-14101) and FIS200913360 , by the ICREA Academia Programme, and by the project PHOCUS within the Future and Emerging Technologies (FET) programme of the EC 7th Framework Programme (grant 240763).
[1] A. S. Pikovsky, M. G. Rosenblum, and J. Kurths, Synchronization, A Universal Concept in Nonlinear Sciences (Cambridge University Press, Cambridge, 2001)

[2] S. Boccaletti, J. Kurths, G. Osipov, D. L. Valladares, and C. S. Zhou, Phys. Rep. 366, 1 (2002).

[3] T. Heil, I. Fischer, W. Elsäßer, J. Mulet, and C. R. Mirasso, Phys. Rev. Lett. 86, 795 (2001).

[4] E. Klein, N. Gross, M. Rosenbluh, W. Kinzel, L. Khaykovich, and I. Kanter, Phys. Rev. E 73, 066214 (2006).

[5] B. B. Zhou and R. Roy, Phys. Rev. E 75, 026205 (2007).

[6] I. Fischer, R. Vicente, J. M. Buldú, M. Peil, C. R. Mirasso, M. C. Torrent, and J. García-Ojalvo, Phys. Rev. Lett. 97, 123902 (2006).

[7] A. S. Landsman and I. B. Schwartz, Phys. Rev. E 75, 026201 (2007).

[8] R. Vicente, C. R. Mirasso, and I. Fischer, Opt. Lett. 32, 403 (2007).

[9] M. Peil, L. Larger, and I. Fischer, Phys. Rev. E 76, 045201(R) (2007).

[10] C. M. Gonzalez, M. C. Torrent, and J. Garcia-Ojalvo, Chaos 17, 033122 (2007).

[11] O. D’Huys, R. Vicente, T. Erneux, J. Danckaert, and I. Fischer, Chaos 18, 037116 (2008).

[12] N. Jiang, W. Pan, B. Luo, L. Yan, S. Xiang, L. Yang, D. Zheng, and N. Li, Phys. Rev. E 81, 066217 (2010).

[13] K. Hicke, O. D’Huys, V. Flunkert, E. Schöll, J. Danckaert, and I. Fischer, Phys. Rev. E 83, 056211 (2011).

[14] J. Tiana-Alsina, J. H. Garcia-Lopez, M. C. Torrent, and J. GarciaOjalvo, Chaos 21, 043102 (2011).

[15] T. Pérez, G. C. Garcia, V. M. Eguíluz, R. Vicente, G. Pipa, and C. Mirasso, PLoS ONE 6, e19900 (2011).

[16] G. D. VanWiggeren and R. Roy, Science 279, 1198 (1998).

[17] A. Sanchez-Diaz, C. R. Mirasso, P. Colet, and P. GraciaFernandez, IEEE J. Quantum Electron. 35, 292 (1999).

[18] A. Argyris, D. Syvridis, L. Larger, V. Annovazzi-Lodi, P. Colet, I. Fischer, J. García-Ojalvo, C. R. Mirasso, L. Pesquera, and K. A. Shore, Nature (London) 438, 343 (2005).
[19] M. C. Soriano, P. Colet, and C. R. Mirasso, IEEE Photon. Technol. Lett. 21, 426 (2009).

[20] E. Ott and J. C. Sommerer, Phys. Lett. A 188, 39 (1994).

[21] P. Ashwin, J. Buescu, and I. Stewart, Phys. Lett. A 193, 126 (1994).

[22] S. C. Venkataramani, B. R. Hunt, and E. Ott, Phys. Rev. E 54, 1346 (1996)

[23] M. Sauer and F. Kaiser, Phys. Lett. A 243, 38 (1998).

[24] C. M. González, C. Masoller, M. C. Torrent, and J. GarcíaOjalvo, Europhys. Lett. 79, 64003 (2007).

[25] V. Flunkert, O. D’Huys, J. Danckaert, I. Fischer, and E. Schöll, Phys. Rev. E 79, 065201(R) (2009).

[26] N. Platt, E. A. Spiegel, and C. Tresser, Phys. Rev. Lett. 70, 279 (1993).

[27] J. F. Heagy, N. Platt, and S. M. Hammel, Phys. Rev. E 49, 1140 (1994).

[28] Y. Nagai and Y. C. Lai, Phys. Rev. E 56, 4031 (1997).

[29] O. D'Huys, R. Vicente, J. Danckaert, and I. Fischer, Chaos 20, 043127 (2010).

[30] J. R. Terry, K. S. Thornburg, D. J. DeShazer, G. D. Vanwiggeren, S. Zhu, P. Ashwin, and R. Roy, Phys. Rev. E 59, 4036 (1999).

[31] D. J. Gauthier and J. C. Bienfang, Phys. Rev. Lett. 77, 1751 (1996).

[32] A. N. Pisarchik, R. Jaimes-Rategui, and J. H. Garcia, Philos. Trans. R. Soc. A 366, 459 (2008).

[33] B. Lading, E. Mosekilde, S. Yanchuk, and Y. Maistrenko, Prog. Theor. Phys. Suppl. 139, 164 (2000).

[34] R. Lang and K. Kobayashi, IEEE J. Quantum Electron. 16, 347 (1980).

[35] J. Mulet, C. R. Mirasso, T. Heil, and I. Fischer, J. Opt. B 6, 97 (2004).

[36] O. D'Huys, I. Fischer, J. Danckaert, and R. Vicente, Phys. Rev. E 83, 046223 (2011).

[37] T. Heil, I. Fischer, and W. Elsäßer, Phys. Rev. A 58, 2672 (1998).

[38] I. Fischer, G. H. M. van Tartwijk, A. M. Levine, W. Elsäßer, E. O. Göbel, and D. Lenstra, Phys. Rev. Lett. 76, 220 (1996).

[39] T. Sano, Phys. Rev. A 50, 2719 (1994). 